\title{
Implementation on a mock-up of the automatic feedback controlled matching options of the full ITER ICRH system
}

\author{
D. Grine, M. Vervier, A. Messiaen, P. Dumortier \\ Laboratory for Plasma Physics \\ Ecole Royale Militaire - Koninklijke Militaire School \\ Avenue de la Renaissance 30, 1000 Brussels
}

The hybrid option has been chosen for the matching of the ITER ICRH system. Presently, the corresponding $3 \mathrm{~dB}$ hybrid matching circuit is being developed, and tested for optimization on a low-powered scaled mock-up. Impedance matching and antenna array current control of the hybrid option is provided by simultaneous feedback control of the decouplers and double stub tuners (23 actuators in total) and is being progressively implemented. Already half of the array has been successfully tested. The article goes into the details of the automatic feedback implementation and covers the developed control systems and algorithms. Feedback stability and starting conditions are also discussed.

Keywords: Load-resilience, hybrid, matching, ITER, ICRF

\section{Introduction}

The ITER ICRH matching system must be resilient, i.e. the magnitude of the reflection coefficient $\Gamma_{\mathrm{G}}$ at the generators must be inferior to $20 \%$, for a wide range of $\mathrm{RF}$ antenna load variations - with respect to the reference load - due to the ELMs and must include strap current control to impose the array radiation spectrum for plasma heating or current drive. The hybrid matching solution alleviates the adverse effects of strong mutual coupling between the heating lines and power sources by the use of decoupler circuits. The CT option [1] was already analyzed and implemented [2], while this paper studies the hybrid option. The main features of the hybrid option are discussed in section 2, while in section 3 , the developed algorithms for decoupling and impedance tuning are presented. In section 4 the implementation on the scaled (1:5) mock-up version of the ITER antenna array located at the ERM/KMS is detailed. The layout of the employed matching circuit is identical to the reference layout for ITER. The antenna array mock-up however is based on the 2003 design [5], which differs in its frequency response at the frequency band edges, but operation in this paper is limited to the central frequency of $45 \mathrm{MHz}$ for which the response is nearly identical. The electrical properties of the 24 strap antenna array and the matching circuit can thus properly be investigated at low power by multiplying the frequency with the inverse of the mock-up scale factor and by placing a movable water tank in front of the antenna array to simulate the plasma loading of the ICRH antenna [4]. For the tuners on the mock-up, we use motor-driven line stretchers. However, for ITER, these can be substituted by other discrete components such as capacitors. The developed algorithms thus remain valid. Similarly, the developed control systems have also been designed with portability in mind. Scaling the power, frequency, and the use of different but equivalent - tuners allow us to obtain a test-bed on which to realistically study the hybrid option. Hence, obtained results allow for extrapolation to the ITER case.

\section{Hybrid matching option}

Fig.1 shows a schematic of the hybrid matching circuit and its control system in our implementation: four $3 \mathrm{~dB}$ hybrids are connected to lines from adjacent poloidal triplets and fed by four power sources with preset phase and same forward power. The layout has been chosen so that the matching circuit with strap current distribution control of the array can be used for both current drive and heating phasing. The locations A$\mathrm{H}$ are preset at voltage anti-node by the $20 \Omega$ lines, as was the case with the CT matching option. When the system is matched, the magnitudes of the voltages at the ports $\mathrm{A}-\mathrm{H}$ are equal and no reflections are present on the heating lines after the double stub tuners (DST). Furthermore, the $3 \mathrm{~dB}$ hybrid splitters impose a $90^{\circ}$ phase shift between the corresponding connected adjacent poloidal lines. Depending on the imposed phasing at the generators, only 7 of the 10 available decouplers are active. Various feedback loops are simultaneously active to bring the circuit to the matched state: (i) feedback on the DSTs (A-H and $\left.\mathrm{A}^{\prime}-\mathrm{H}^{\prime}\right)$ to minimize reflections and (ii) feedback on the poloidal decouplers and either the toroidal ones (case of current drive phasing) or the toroidal-poloidal ones (case of heating phasing) to control the amplitude at the voltage anti-node points A-H while maintaining the mutual coupling at a low level [1] [3]. In total, 26 tuners are implemented in the circuit and 23 of these are active for a given phasing. Each tuner has its own feedback loop driven by a software-based controller as shown in Fig.1 using specific algorithms for the decoupling and impedance matching. A view of the mock-up with its main components is shown in Fig.2.

\section{Algorithms}

\subsection{Decoupling}

All decouplers have the same structure [2] and are controlled using the same algorithm. The length of the shorted stub between the two $\lambda_{0} / 4$ lines is controlled by an error signal proportional to the difference in 
magnitude of the two voltages $\mathrm{V}_{1}$ and $\mathrm{V}_{2}$ at the ports of the decoupler. In the $\mathrm{CT}$ matching solution, the decouplers only minimize the mutual coupling between the heating lines, whereas in the hybrid matching solution a double purpose is fulfilled simultaneously: (i) minimization of the coupling between the lines and (ii) control of the magnitude of the voltages at the ports A-H to control the current distribution in the antenna straps. The latter is possible due to the excellent correlation between the two, both in magnitude as well as in phase.

3dB hybrid matching circuit

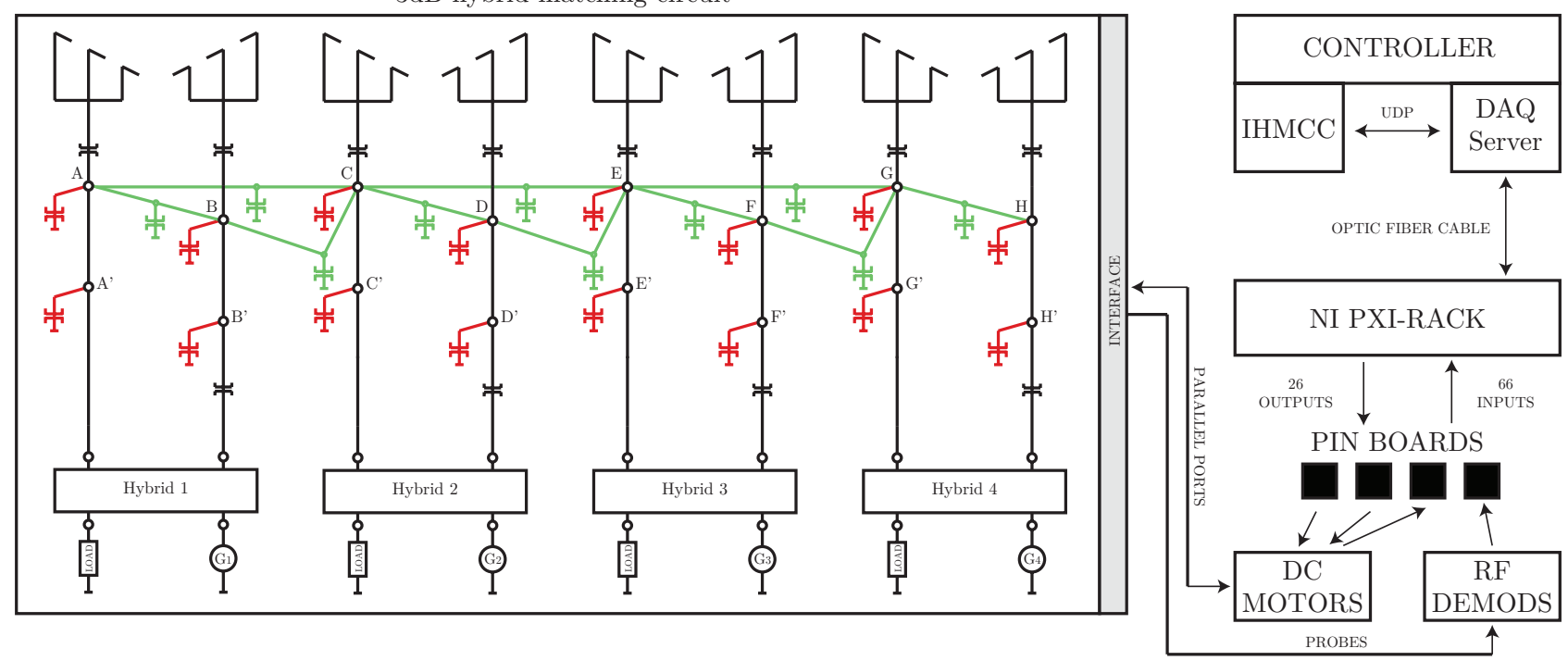

Fig.1 Implementation of the 3dB hybrid matching circuit. Left: 10 decouplers (green) between the ports A-H and 16 matching stubs (red) on the 8 heating lines; Right: feedback system with software-based controller and associated hardware

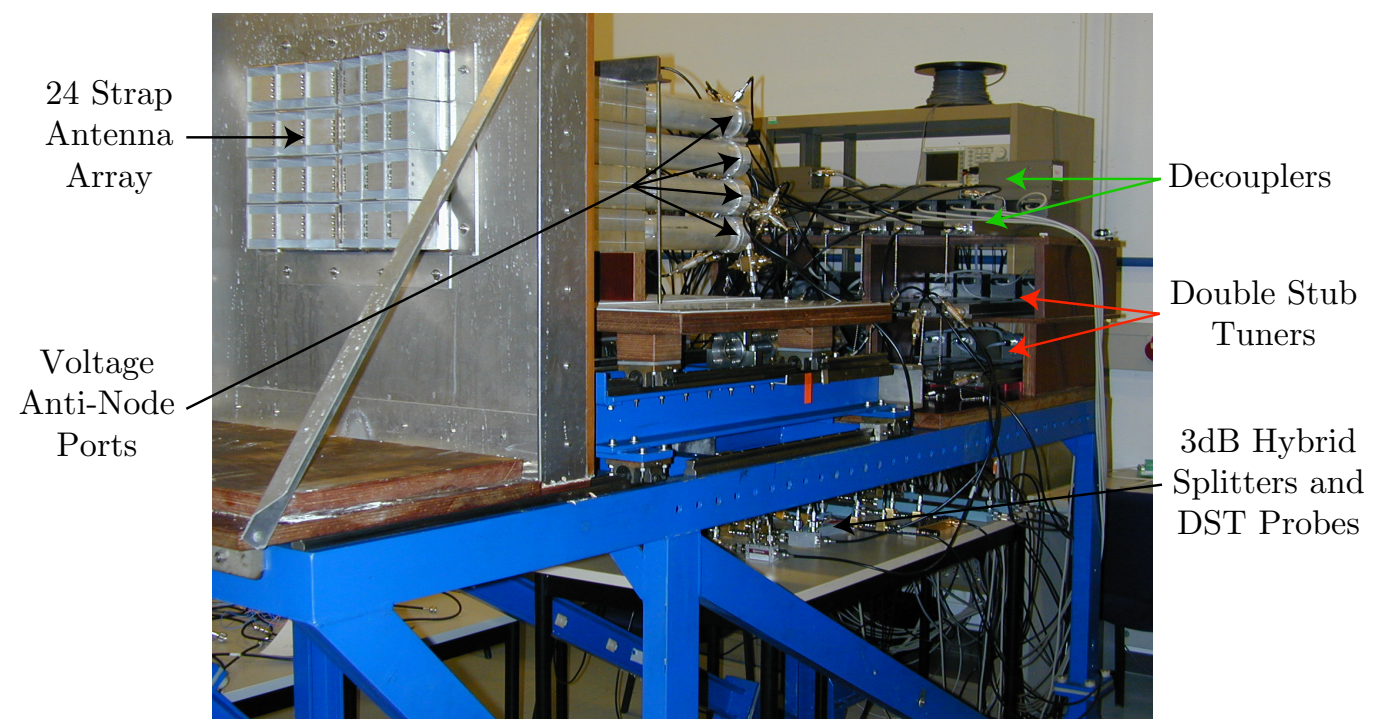

Fig.2 Mock-up of the ITER antenna and the 3dB hybrid matching circuit

\subsection{Impedance matching}

Various algorithms were developed for the automatic impedance matching. We discuss those most of interest.

\section{Bang-Bang}

In the Bang-Bang algorithm, the two stubs in a DST are controlled by identical state-machines running in parallel and only differing in their initial state, received events and emitted actions. The length of the first stub is initially increased, after which the magnitude of the reflection coefficient $\Gamma_{\mathrm{HO}}$ after the DST (at the hybrid outputs) is measured and evaluated. If the increase resulted in a lower $\left|\Gamma_{\mathrm{HO}}\right|$, the line length will continue to increase. However, if a higher $\left|\Gamma_{\mathrm{HO}}\right|$ is measured, the line will decrease. This sequence is repeated until both directions result in an increase of $\left|\Gamma_{\mathrm{HO}}\right|$, after which the active tuning stub is put in the "Idle" state and an activation signal is emitted to the second tuning stub. The Bang-Bang algorithm requires only a single measurement and is very robust: convergence - if possible - is guaranteed. However, it is a very slow algorithm, since only one tuning stub is active at a time. 


\section{Fast Bang-Bang}

To address the shortcoming of the Bang-Bang algorithm, the two parallel state-machines controlling the individual stubs are brought together in a single statemachine controlling both stubs simultaneously, resulting in a state for each possible situation. Transitions between these states for each step $n$ are based on the evaluation of the Boolean function $b(n)=\left|\Gamma_{H O}(n)\right|<\left|\Gamma_{H O}(n-1)\right|$. Since the states in this sequence are combinations of the individual states of the Bang-Bang algorithm, the lengths of the two stubs are adjusted simultaneously, resulting in significantly faster convergence. However, if the decouplers in the matching circuit are far off their solutions, the mutual coupling between different DSTs is considerably large, and the increase in speed is almost entirely lost due to the inability of the algorithm to sufficiently distinguish between the effects on the reflection coefficient of the various tuning stubs. This increases the number of wrong decisions in the sequence diagram and results in lost time before the algorithm realizes it has made a mistake.

\section{Real/Imag}

Mitigating the problems of the Bang-Bang and Fast Bang-Bang algorithms can be done by analyzing the movements of the input admittances of the DSTs on the Smith Chart as it reveals interesting relations between the two stubs of a single DST and their effect on the measured $\Gamma_{\mathrm{HO}}$ at the generator-side of the second stub of the DST. Indeed, the first stub influences both the real and imaginary parts, while the second stub influences only the imaginary part. Linearizing $\Gamma_{\mathrm{HO}}$ around the matching point yields stub error signals that are function of the real and imaginary parts of the measured reflection coefficient. It is thus no longer sufficient to measure the magnitude; the phase of $\Gamma_{\mathrm{HO}}$ must also be known. Additional voltage probes are placed on the lines after the DSTs to estimate the phase and write the error signals as a function of these measurements. The basic assumption of the algorithm, i.e. the valid linearization around $\Gamma_{\mathrm{HO}}=0$, is very similar to techniques used for the internal CT matching of the ILA at JET, and the CT matching at TEXTOR [6]. The Real/Imag algorithm provides robust impedance matching with relatively short convergence times. It is not minimum-phase, but exhibits good transient behavior when load variations occur with respect to the matched state. Therefore, it is the preferred algorithm for DST control in the $3 \mathrm{~dB}$ hybrid matching circuit.

\section{Implementation}

\subsection{Mock-up}

The tuners are commercial motor-driven line stretchers with shorted terminations on one of their ports. They have a limited range that covers $20 \%$ of the wavelength and are equipped with parallel ports that allow the line length to be adjusted by applying a DC voltage to the custom-made DC motors that are connected to the appropriate pins of the parallel ports on one side, and to the pin boards of the National Instruments (NI) PXI rack on the other. Pulse width modulation is used to control the relative velocities of the tuners as the dynamic behavior differs (e.g. different velocities for the same input voltage, different activation thresholds, etc.). The tuners always move at maximum velocity for both directions and on average adjust the lines at a rate of $10.9 \mathrm{~mm} / \mathrm{s}$, which is slow since the wavelength at the scaled frequency for the mock-up corresponds to $1.43 \mathrm{~m}$. In practice, tuners move much faster, e.g. at TEXTOR, capacitors move at $1.3 \mathrm{pF} / \mathrm{ms}$ [6]. Finally, custom-made RF demodulators demodulate the circuit measurements.

\subsection{Control systems}

A software-based controller steers the automatic feedback. It is mainly written in $\mathrm{C}++$, with the exception of the low-level hardware portions of the code, which are written in C. The code is cross-platform compatible, highly portable, modular and faster than previous implementations developed for the CT solution in [2]. Communication with external modules is possible via the User Datagram Protocol (UDP), allowing not only for remote control of the feedback systems, but also for extensive testing and validation of the control systems prior to implementation. The software-controller is divided in two core components: IHMCC (ITER 3dB Hybrid Matching Circuit Controller) and DAQ Server (Data Acquisition Server). Both applications are multithreaded: various tasks run in concurrence. IHMCC runs the algorithms, a UDP client/server, a data recorder and a GUI. It is entirely independent of the physical implementation of the matching circuit, yet solely responsible for correctly piloting the tuner susceptances. DAQ Server is in charge of the physical control of voltages applied to and retrieved from line stretchers, probes, etc. It runs a UDP client/server, a GUI, and the hardware interface code. External libraries $Q t$ and $N I$ DAQmx facilitate specific programming aspects. The clear division of tasks allows for DAQ Server to be substituted by a simulation code called SimServer without altering IHMCC - in order to validate the control system. SimServer - written in Matlab and Java - listens on the UDP broadcasting port of IHMCC and translates the 26 steering voltages destined for the DAQ hardware into movements of the shorted stubs in the transmission line model of the circuit. This, along with the measured scattering matrix at the ports $\mathrm{A}-\mathrm{H}$ on the mock-up, allows us to simulate the full $3 \mathrm{~dB}$ hybrid matching circuit - including practical limitations on the range of component, etc. - and broadcast the resulting 66 measurements to the UDP listening port of IHMCC. This enables us to validate the control system prior to implementation on the mock-up. In our setup, IHMCC and SimServer run on an OS $\mathrm{X}$ or Linux machine and DAQ Server on a Linux machine connected to the PXI rack via optical fiber. Communication is done via RJ-45cable or remotely via the local network. Finally, note that when porting this implementation to other practical setups, only the data interfacing portions of DAQ Server require modification. Everything else remains unaltered, due to the introduced abstraction level. 

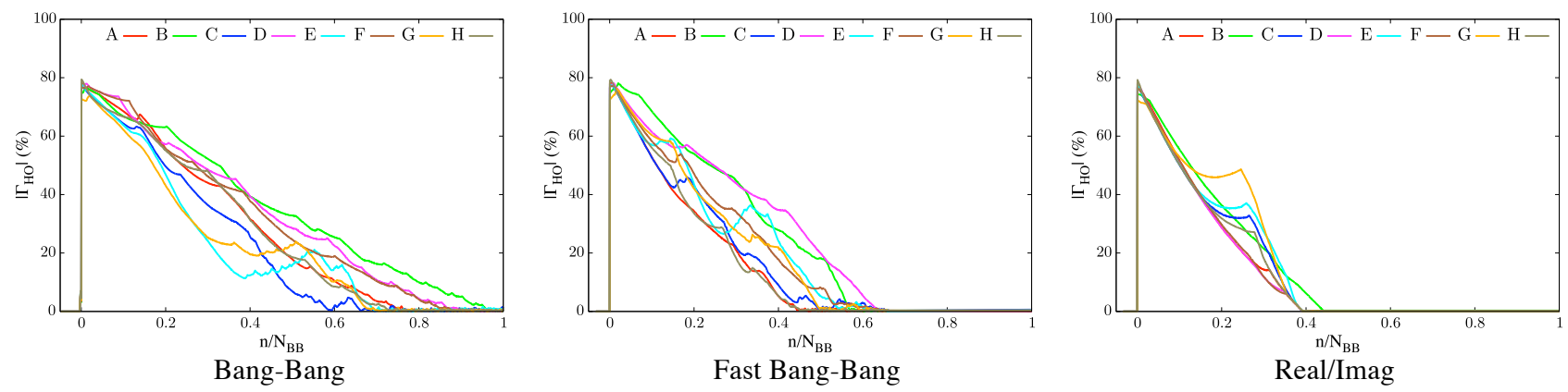

Fig.3 Performance comparison for $\mathrm{R}_{\text {aeff }}$ excursion from $2.25 \Omega / \mathrm{m}$ to $5 \Omega / \mathrm{m}$ and current drive. $\mid \Gamma_{\mathrm{HO}} \mathrm{l}$ for the heating lines A-H as a function of the normalized iterations $\mathrm{n} / \mathrm{N}_{\mathrm{BB}}$, where $\mathrm{N}_{\mathrm{BB}}$ is the number of iterations required for the Bang-Bang algorithm to converge
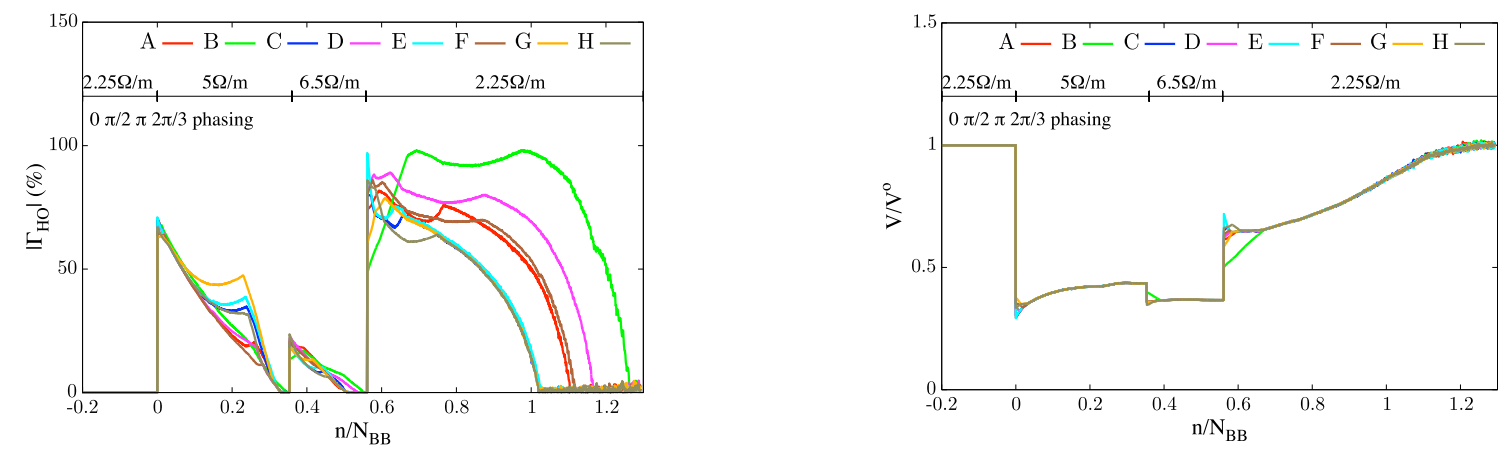

Fig.4 Transient simulation of consecutive $\mathrm{R}_{\mathrm{a}, \text { ff }}$ excursions and current drive using the Real/Imag algorithm: $\left|\Gamma_{\mathrm{HO}}\right|$ for the heating lines A-H (left), and voltage magnitude at the ports A-H normalized to the initial voltage (right)

\section{Results}

Fig.3 and Fig.4 are simulations of IHMCC, using SimServer, for the full circuit. Fig. 3 compares algorithm performance, with simultaneous decoupling, and confirms previously discussed algorithm characteristics. Algorithms with better convergence than the Real/Imag algorithm are still investigated, but high mutual coupling complicates development of minimum-phase algorithms. Iterations in the plots are normalized to the number of iterations $\mathrm{N}_{\mathrm{BB}}$ required for the Bang-Bang algorithm to converge after an excursion from $2.25 \Omega / \mathrm{m}$ to $5 \Omega / \mathrm{m}$ during current drive. As previously mentioned, the tuner velocities are very slow. On the mock-up, $n=N_{B B}$ corresponds on average to $125 \mathrm{~s}$. Since all tuners move at maximum velocity, extrapolation for convergence times on ITER only depends on the dynamic properties of the employed tuners. Fig.4 (a) shows $\left|\Gamma_{\mathrm{HO}}\right|$ for a series of consecutive load variations expressed by the distributed effective antenna resistance $R_{a, \text { ff }}$ (b) shows the magnitude of the normalized voltages at the ports A-H. Matching is achieved even for large excursions and similar results are obtained for heating phasings. Interesting findings concerning the stability of the system were made. Observations show that convergence heavily depends on the starting positions, i.e. initial line stretcher lengths. When starting in a matched state, all algorithms converge after a load excursion. However, when positioning the line lengths randomly, convergence is no longer guaranteed. The relative velocities of the line stretchers must then be tuned: DSTs are set to move faster than decouplers because the latter require smaller adjustments. This is similar to the empirical tuning of PID-coefficients in previous implementations. In practice, initial positions are set near the optimal positions for vacuum. This corresponds to removing the water load in front of the antenna. When moving the load back, the algorithms then converge because line lengths are in the vicinity of these optimal lengths.

In conclusion: (i) the automatic feedback on the 23 active actuators is capable of tracking load variations in $\mathrm{R}_{\mathrm{a}, \mathrm{eff}}$, both in terms of the reflections as in terms of the current distribution in the antenna straps, and (ii) the developed control systems and algorithms are suitable, but dependence on initial line lengths must be taken into account for stability.

\section{References}

[1] A. Messiaen et al., Nuclear Fusion 49 (2009).

[2] D. Grine et al., Tests on a mock-up of the feedback controlled matching options for the ITER ICRH system, Proc. of the $18^{\text {th }}$ top. conf. on RF power in plasmas (2009)

[3] M. Vervier et al., Analysis of the ITER ICRH decoupling and matching system, Proc. of the $18^{\text {th }}$ top. conf. on RF power in plasmas (2009)

[4] A. Messiaen et al., Realization of a test facility for the ITER ICRH antenna plug-in by means of a mock-up with salted water load, Fusion engineering and design (2004)

[5] Study of the ITER ICRH system with external matching by means of a mock-up loaded by a movable waterload, Nuclear Fusion 46 (2006)

[6] F. Durodié, M. Vervier, Design of an automatic matching device for TEXTOR's ICRH system, Proc. Europhys. top. conf. on RF heating and current drive of fusion devices, 16E (1992) 